\title{
Speaking from the Inside: Challenges Faced by Communication Researchers Investigating Disease-Related Issues in a Hospital Setting
}

\author{
Céline Bourquin • Friedrich Stiefel • Pascal Singy
}

Published online: 18 January 2015

(C) Springer Science+Business Media New York 2015

\begin{abstract}
This commentary came from within the framework of integrating the humanities in medicine and from accompanying research on disease-related issues by teams involving clinicians and researchers in medical humanities. The purpose is to reflect on the challenges faced by researchers when conducting emotionally laden research and on how they impact observations and subsequent research findings. This commentary is furthermore a call to action since it promotes the institutionalization of a supportive context for medical humanities researchers who have not been trained to cope with sensitive medical topics in research. To that end, concrete recommendations regarding training and supervision were formulated.
\end{abstract}

Keywords Medical humanities · Emotionally laden research · Disease-related issues · Communication

The integration of the humanities in medicine (Petersen et al. 2008; Ousager and Johannessen 2010; Doukas et al. 2012) has resulted both in teachings for medical students and in research on health- and disease-related issues by interdisciplinary teams involving clinicians and researchers/teachers in medical humanities.

Several articles on qualitative research and particularly on qualitative health research have addressed the personal and emotional impact that such research potentially has on the researchers dealing with emotionally laden issues (Dunn 1991; Rowling 1999; Chesney 2001; Gilbert 2001; Perry et al. 2004; Rager 2005a, b; Dickson-Swift et al. 2008; Woodby et al. 2011), emphasizing the vulnerability of the observer-researcher, the threat to his/her emotional well-being and health, the physical and emotional symptoms experienced, and the "compassion stress" around certain interviewees. Given this general background, this reflection paper aims more precisely to examine how interdisciplinary research conducted in the medical setting may affect researchers in medical humanities, their research outcomes, and ultimately - by translation of the research outcomes into clinical practice - the patient-health care provider interaction.

C. Bourquin $(\bowtie) \cdot$ F. Stiefel $\cdot$ P. Singy

Psychiatric Liaison Service, Lausanne University Hospital, Les Allières, 1011 Lausanne, CHUV,

Switzerland

e-mail: celine.bourquin@chuv.ch 
Based on the experience of researchers in communication (sociolinguists) working in the psychiatric liaison service of a university hospital, this paper reflects mainly on the personal and emotional challenges faced by researchers when conducting emotionally laden research on disease-related issues and on how the challenges encountered potentially impact their observations and subsequent research findings. The focus will be, at the same time, on the fact of being a communication researcher in a medical service. Subsequent to this reflection, concrete practical recommendations are proposed to diminish the emotional burden of research, to improve researchers' coping and self-care strategies, and to strengthen research outcomes.

Although the reflection and the proposed recommendations focus on researchers in communication, they obviously concern as well researchers in other disciplines within the developing field of medical humanities such as sociology, anthropology and history.

\section{Being a communication researcher in a psychiatric liaison service}

Over the last ten years, the Psychiatric Liaison Service of the Lausanne University Hospital (Switzerland) has developed a Human Sciences Unit consisting of a team of sociolinguists investigating the patient-clinician relationship and medical communication. Members of this Unit have conducted or participated in interdisciplinary research projects focusing among others on communication (i) in the HIV/AIDS context (e.g., the understanding and misunderstanding of prevention messages in the general population); (ii) in cancer care (e.g., assessment of medical student and clinician communication skills); and (iii) in end-of-life care (e.g., development of a Communication Skills Training for clinicians who care for patients suffering from a progressive and life-threatening disease). Since this Human Sciences Unit is integrated in a medical setting, its members interact daily with clinicians (especially with psychiatrists and psychologists), come across patients and visitors in waiting rooms and hospital corridors, take part in the twice-monthly clinical and scientific seminars of the service, and network with health care providers. Therefore, researchers of this Human Sciences Unit are, as non-health care providers, in a kind of "observer third party" position, which allows a continuous observation of both the medical system and the relationship and communication patterns between clinicians and patients and among health care providers.

This implies that the researchers face emotionally laden issues related to their research and to the fact of being immersed in a hospital setting. Even though they work in a "containing context" (a psychiatric service in a university hospital), members of the Human Sciences Unit - like many humanities researchers - have neither been trained to cope with emotionally laden issues, as disease-related ones may be, nor are they accustomed to discussing their experiences. Indeed, experiencing emotions in research may still be considered by researchers as a lack of objectivity, professionalism and/or competence or as self-centered, which can as a result hamper any expression and discussion. However, the lack of expression does not mean that researchers' emotions facing disease-related suffering and the hospital setting are non-existent; on the contrary, psychological distress may even be enhanced due to the fact that it is not addressed, expressed and reflected.

\section{Particular challenges faced by researchers investigating disease-related issues}

When conducting research on disease-related issues, such as HIV/AIDS, cancer, and death and dying, communication researchers may for the first time face their own potentially risk behaviors, health vulnerabilities, and death. They thus have to cope with more or less 
conscious anxieties and stress related to these issues. Furthermore, research in the HIV/AIDS context, using semi-structured interviews, or research on breaking bad news in oncology, based on the analysis of videotaped interviews with simulated or actual patients, may have various effects on the researcher. Among others, such research can cause resonating effects (other's/patient's emotions and concerns trigger emotions and concerns in the researcher), collusion (other/patient and researcher struggle with the same unconscious and unresolved problems), projection (the researcher projects his own emotions on the research subject), identification (e.g., in cases when there are cross-identifiers such as patient/interviewee of the same age as the researcher), and interference with bodily signs or symptoms experienced by the researcher.

As a result, researchers may react with the misinterpretation of their own bodily signs as the symptoms of disease reported by (simulated) patients in the interviews that they analyze, with (increased) fear of becoming ill, and with hypochondria, anxiety and emotional distress. In addition, research on end-of-life issues confronts the researchers with their own representations of and attitudes towards death and dying and potential death-related fears and experiences. This might constitute a stressor as well as a distressor for them.

The abovementioned effects and psychological challenges are obviously not specific to communication researchers or medical humanities researchers. They affect to a much greater extent the health care providers who actually interact with and care for patients. The psychological challenges faced, for instance, by physicians and nurses (especially in oncology) and residents are well documented (Ramirez et al. 1996; Shanafelt et al. 2002, 2006; Fallowfield and Jenkins 2004; Stiefel and Krenz 2012).

Because of a defensive attitude, a physician may lack empathy and attention to respond to patient's needs, and the interpersonal relationship with the patient may be affected. The same is not true for the researchers who are not involved in clinical care. However, it seems important to consider the psychological challenges encountered by communication researchers not only in isolation - the researcher is still (too) often viewed as an instrument through which data are produced and analyzed, but also in relation to the influence they potentially have on the approach of communication researchers to the medical interaction.

When based on an observational approach of patient-health care provider communication behaviors, communication research largely relies on the researcher's perception or assessment. This may be influenced by how researchers cope with emotionally laden interactions and may notably result in the inadvertent missing of certain elements, avoidance behaviors when observing certain issues, and data misinterpretation.

An observational instrument such as the well-known RIAS (Roter Interaction Analysis System) (2006), for example, allows, with its tailored categories reflecting the content and form of the medical interaction, a relatively objective coding, insofar as it also involves the researcher's intuition regarding the interpretation of how things are said from a tonal point of view. Communication researchers facing distressing interviews could tend to categorize, according to how they cope with the interaction context, certain utterances in affect categories (e.g., statements of disapproval or complaint, statements of concern or worry) or as neutral task-oriented statements (e.g., statements of information). In this respect, double-coding is used to decrease "misintuitions" and misinterpretations by researchers although a priori without having any impact on the emotional burden of research.

These particular challenges faced by researchers seem not to be specific to qualitative research. Indeed, we assume that quantitative research, for example, when requiring analyses of large body of transcripts or videos of medical interactions between clinicians and cancer patients (actual or simulated) by researchers (vs. computerized coding), can have similar effects on them and be just as much personally and emotionally demanding. 


\section{Recommendations for researchers investigating sensitive medical topics}

There is a constant interplay among the personal experiences, the emotions and the thought processes that researchers such as communication researchers have to manage in order to reach a balance between involvement and detachment in research. This balance or equilibrium, when studying sensitive medical topics seems difficult to achieve without preparatory training and support, and this may lead to emotional exhaustion and concomitant under-involvement or detachment with regard to the research. While researchers may have support from their social network throughout the process, at least if their network feels disposed to have recurring discussion about disease- and death-related issues (which cannot be taken for granted), such private support cannot be as effective as, for instance, regular peer and professional support.

Guidelines on health research especially promote training and education (to increase awareness of the potentially emotional nature of research) and research debriefing and supervision (Dunn 1991; Rager 2005a, b; Dickson-Swift et al. 2008; Woodby et al. 2011). However, our sense is that these guidelines are not formulated in such a way as to allow concrete application to communication research practice. Still, based on existing literature and related calls for improved preparation and support of researchers dealing with sensitive topics as well as our own experience in the health field (CB and PS are sociolinguists and FS a liaison psychiatrist), we expect that present and future communication researchers in the medical setting would specifically benefit from (i) education and training addressing the challenges of investigating emotionally laden topics, (ii) peer debriefing sessions, and (iii) professional supervision.

Undergraduate and postgraduate training of students and young researchers should prepare them to deal and cope with sensitive topics in research. Training could focus not only on research on the physician/patient population but also, among others, on groups at the margins of society (e.g., injecting drug users (IDUs), undocumented migrants) and related sensitive issues. Teaching could be provided by experienced researchers in the humanities who have already dealt with emotionally laden topics, together with liaison psychiatrists or psychologists, who are competent to address the psychological challenges of conducting such research.

With regard to researchers already involved in investigating disease-related topics, room for peer (co-researcher) debriefing sessions should be included in research timetables and provided during research processes. Such sessions might allow researchers (a) to identify and express their own emotions, (b) to share possible ways to cope with them, and to collectively identify (c) stressors and distressors, (d) strategies to handle them more effectively, and (e) potential effects on analyses and outcomes.

Group supervisions, occasional and/or upon request of the researchers, by research supervisors could be an opportunity for researchers to increase the identification of their own difficulties, to understand underlying mechanisms, and to develop strategies for stress management. Group supervisions could be inspired by the widely used Balint group method (Balint 1964; Merenstein and Chillag 1999), i.e., discussion groups intended for health care providers (e.g., residents, practicing physicians, nurses) led by trained facilitators with the aim of identifying, understanding and coping with challenging clinical situations.

After all, supervision of the health care providers has a positive impact both on the providers themselves and on establishing a good interpersonal relationship with patients. With regard to communication researchers, we can expect that the institutionalization of a supportive context will result especially in strengthening research findings that aim, by translation into clinical practice, at improving the patient-health care provider relationship from an interpersonal and communication point of view. 


\section{Conclusion}

This reflection as well as the proposed recommendations do not aim to "abrade" emotions experienced by the researchers or to reduce them to a problem to be overcome. Emotions are an important source of information, both for the researcher and for the topic under investigation; the aim of the recommendations is to help the researcher to deal with a potential negative impact on him/her and on the quality of the research. It is therefore an attempt to include the researcher's emotions in a thoughtful and constructive way as a part of the research and analysis process. In other words, the challenge is to get the "adequate" distance or balance that allows communication researchers in medicine to remain sensitive and to some extent empathetic, to strengthen their observer gaze, and to thus increase the quality of their research, their well-being and their job satisfaction.

\section{References}

Balint, M. 1964. The Doctor, his Patient and the Illness. Madison: International University Press.

Chesney, M. 2001. "Dilemmas of Self in the Method." Qualitative Health Research 11:127-135.

Dickson-Swift, V., E.L. James, S. Kippen, and P. Liamputtong. 2008. "Risk to Researchers in Qualitative Research on Sensitive Topics: Issues and Strategies." Qualitative Health Research 18:133-144.

Doukas, D.J., L.B. McCullough, and S. Wear. 2012. "Medical Education in Medical Ethics and Humanities as the Foundation for Developing Medical Professionalism." Academic Medicine 87:334-341.

Dunn, L. 1991. "Research Alert! Qualitative Research may be Hazardous to your Health!" Qualitative Health Research 1:388-392.

Fallowfield, L., and V. Jenkins. 2004. "Communicating Sad, Bad and Difficult News in Medicine." Lancet 363: $312-319$.

Gilbert, K.R., ed. 2001. The Emotional Nature of Qualitative Research. London: CRC Press.

Merenstein, J.H., and K. Chillag. 1999. “Balint Seminar Leaders: What Do They Do?” Family Medicine 31:182186.

Ousager, J., and H. Johannessen. 2010. "Humanities in Undergraduate Medical Education: A Literature Review." Academic Medicine 85:988-998.

Perry, C., M. Thurston, and K. Green. 2004. "Involvement and Detachment in Researching Sexuality: Reflections on the Process of Semistructured Interviewing." Qualitative Health Research 14:135-148.

Petersen, A., A. Bleakley, R. Brömer, and R. Marshall. 2008. "The Medical Humanities Today: Humane Health Care or Tool of Governance." Journal of Medical Humanities 29:1-4.

Rager, K.B. 2005a. "Compassion Stress and the Qualitative Researcher." Qualitative Health Research 15:423430.

- 2005b. "Self-care and the Qualitative Researcher: When Collecting Data can Break your Heart." Educational Researcher 34:23-27.

Ramirez, A.J., J. Graham, M.A. Richards, A. Cull, and W.M. Gregory. 1996. "Mental Health of Hospital Consultants: The Effects of Stress and Satisfaction at Work." Lancet 347:724-728.

Roter, D. 2006. The Roter Method of Interaction Process Analysis. RIAS Manual. Baltimore: John Hopkins University.

Rowling, L. 1999. "Being in, Being out, Being with: Affect and the Role of the Qualitative Researcher in Loss and Grief Research." Mortality 4:167-181.

Shanafelt, T.D., K.A. Bradley, J.E. Wipf, and A.L. Back. 2002. "Burnout and Self-reported Patient Care in an Internal Medicine Residency Program.” Annals of Internal Medicine 136:358-367.

Shanafelt, T.D., H. Chung, H. White, and L.J. Lyckholm. 2006. "Shaping your Career to Maximize Personal Satisfaction in the Practice of Oncology." Journal of Clinical Oncology 24:4020-4026.

Stiefel, F., and S. Krenz. 2012. "Psychological Challenges for the Oncology Clinician who has to Break Bad News." In New Challenges in Communication with Cancer patients, edited by A. Surbone, M. Zwitter, M. Rajer, and R. Stiefel. Berlin: Springer.

Woodby, L.L., B.R. Williams, A.R. Wittich, K.L. Burgio. 2011. "Expanding the Notion of Researcher Distress: The Cumulative Effects of Coding." Qualitative Health Research 21:830-838. 